\section{Bloqueo regional genital en urología}

Carrillo-Córdova LD, ${ }^{1}$ Carrillo-Córdova JR, ${ }^{2}$ Villena-López EL, ${ }^{3}$ Carrillo-Esper $\mathrm{R}^{4}$

\section{Resumen}

El bloqueo regional genital en urología ofrece ventajas como: menor tiempo de estancia hospitalaria y de complicaciones relacionadas con la técnica anestésica, mejor control del dolor peri y posoperatorio y disminución de costos asociados con la cirugía. Este tipo de bloqueo se utiliza para efectuar diversos procedimientos quirúrgicos en los genitales, principalmente: circuncisión, meatotomía, corrección de la enfermedad de Peyronie, incisión dorsal del prepucio, inserción de prótesis del pene, cirugía escrotal y testicular, hidrocelectomía, quiste de epidídimo, vasectomía, varicocelectomía, biopsia testicular, orquiectomía y orquidopexia. Esta revisión describe las técnicas de bloqueo genital en urología.

PALABRAS CLAVE: bloqueo regional genital, bloqueo del pene, bloqueo en urología.
Rev Mex Urol. 2017 Nov-Dec;77(6):492-499.

\section{Genital regional anesthesia in urology}

Carrillo-Córdova LD, ${ }^{1}$ Carrillo-Córdova JR, ${ }^{2}$ Villena-López EL, ${ }^{3}$ Carrillo-Esper $\mathrm{R}^{4}$

\begin{abstract}
In urologic procedures, genital regional block offers many advantages, such as shorter hospital stay, fewer anesthesia-related complications, good perioperative and postoperative pain control, and reduced surgery-related costs. This type of blockade has been used for numerous surgical procedures involving the genitalia, including circumcision, meatotomy, correction of Peyronie's disease, dorsal incision of the prepuce, penile prosthesis insertion, scrotal and testicular surgery, hydrocelectomy, epididymal cyst, vasectomy, varicocelectomy, testicular biopsy, orchiectomy, and orchidopexy. The present review describes the regional anesthesia techniques used in urologic procedures involving the genitalia.
\end{abstract}

KEYWORDS: Genital block; Penile block; Blockade in urology
${ }^{1}$ Departamento de Urología, Hospital General de México Dr. Eduardo Liceaga, Ciudad de México. 2 Departamento de Cirugía Plástica y Reconstructiva, Hospital General Dr. Manuel Gea González, Ciudad de México.

${ }^{3}$ Departamento de Medicina Interna, Hospital Ángeles del Pedregal, Ciudad de México.

${ }^{4}$ Jefe de la División de Áreas Críticas, CENIAC, Instituto Nacional de Rehabilitación, Ciudad de México.

Recibido: abril 2017

Aceptado: septiembre 2017

Correspondencia

Luis Daniel Carrillo Córdova

carrillocor@gmail.com

Este artículo debe citarse como

Carrillo-Córdova LD, Carrillo-Córdova JR, Villena-López EL, Carrillo-Esper R. Bloqueo regional genital en urología. Rev Mex Urol. 2017 nov-dic;77(6):492-499. DOI: https://doi.org/10.24245/revmexurol.v77i6.1403 


\section{ANTECEDENTES}

El advenimiento de las técnicas de bloqueo regional ha permitido al urólogo realizar diversos procedimientos sin exponer al paciente a los efectos colaterales de la anestesia general. Los principales anestésicos administrados para este tipo de procedimientos son la lidocaína y bupivacaína (Cuadro 1).

Aunado con lo anterior, puede evitar la manifestación de síntomas asociados con el posoperatorio, como: náusea, vómito, complicaciones provocadas por bloqueos espinales y retención urinaria, lo que permite al paciente una deambulación más rápida, tolerancia a la dieta, egreso hospitalario temprano y disminución de los costos por hospitalización.

El objetivo de este artículo es describir las principales técnicas de bloqueo regional en urología, que pueden realizarse en un quirófano ambulatorio, en la sala de urgencias o en cualquier quirófano completamente equipado.

La selección de pacientes representa un punto clave para implementar las diferentes técnicas de anestesia. Éstos deben aceptar el procedimiento sugerido $y$, preferentemente, tratarse de sujetos poco ansiosos. La aplicación de anestesia regional debe administrarla un anestesiólogo certificado, con la finalidad de disminuir las molestias y complicaciones. El objetivo princi-

Cuadro 1. Comparación entre lidocaína y bupivacaína

\begin{tabular}{|l|c|c|}
\hline & Lidocaína & Bupivacaína \\
\hline Dosis & $3 \mathrm{mg} / \mathrm{kg}$ & $2 \mathrm{mg} / \mathrm{kg}$ \\
\hline Dosis máxima & $200 \mathrm{mg}$ & $150 \mathrm{mg}$ \\
\hline Dosis con epinefrina & $6 \mathrm{mg} / \mathrm{kg}$ & $2 \mathrm{mg} / \mathrm{kg}$ \\
\hline Dosis máxima con epinefrina & $500 \mathrm{mg}$ & $150 \mathrm{mg}$ \\
\hline Tiempo de acción & $2-10 \mathrm{~min}$ & $30-40 \mathrm{~min}$ \\
\hline Duración & $60-180 \mathrm{~min}$ & $2-3$ horas \\
\hline
\end{tabular}

pal del procedimiento es mantener al paciente cómodo, despierto y consciente.

\section{Bloqueo del pene}

La administración de anestesia local en procedimientos quirúrgicos del pene incluye: tratamiento de la parafimosis, incisión dorsal del prepucio, meatotomía, inserción de prótesis, corrección de la enfermedad de Peyronie, procedimientos vasculares y circuncisión ${ }^{1-4}$

\section{(Cuadro 2).}

Hsu y sus colaboradores reportaron una serie de 21 pacientes con disfunción eréctil, intervenidos de prótesis de pene con anestesia regional, mediante bloqueo del nervio pudendo, infiltración peripeneana y bloqueo crural. Con esta técnica obtuvieron excelentes resultados y entre las complicaciones tempranas reportaron: dolor local, equimosis subcutánea, hematoma local y punción de vasos. No registraron complicaciones tardías. ${ }^{5}$

Otro estudio efectuado por los mismos autores, en el que intervinieron a 29 pacientes para la corrección quirúrgica de la enfermedad de Peyronie, a quienes colocaron un injerto venoso, reportaron buenos resultados, con las mismas complicaciones tempranas que en su serie de prótesis de pene; por tanto, concluyeron que la

Cuadro 2. Procedimientos en los que se administra anestesia regional en el pene con éxito

\begin{tabular}{|l|l|}
\hline Procedimiento & Tipo de anestesia \\
\hline Circuncisión & $\begin{array}{l}\text { Bloqueo dorsal, infiltración } \\
\text { local, lidocaína-epinefrina }\end{array}$ \\
\hline Meatotomía & $\begin{array}{l}\text { Bloqueo dorsal, infiltración } \\
\text { local del frenillo }\end{array}$ \\
\hline $\begin{array}{l}\text { Corrección de enfermedad } \\
\text { de Peyronie }\end{array}$ & $\begin{array}{l}\text { Bloqueo dorsal, infiltración } \\
\text { local }\end{array}$ \\
\hline Incisión dorsal del prepucio & $\begin{array}{l}\text { Bloqueo dorsal, infiltración } \\
\text { local }\end{array}$ \\
\hline Inserción de prótesis de pene & $\begin{array}{l}\text { Bloqueo dorsal, infiltración } \\
\text { local }\end{array}$ \\
\hline
\end{tabular}


anestesia regional del pene tiene baja morbilidad, menos efectos adversos de la anestesia local y regreso más rápido a las actividades cotidianas. ${ }^{6}$

Una serie de 89 pacientes intervenidos para circuncisión reportó que la anestesia local en el pene es una técnica sencilla, con excelente analgesia y menos complicaciones. ${ }^{7}$

Aún se discute la administración de lidocaína con epinefrina para el bloqueo del pene. Schnabl y su grupo evaluaron esta combinación y no reportaron ningún efecto adverso en la vascularidad del pene, ni problemas relacionados con el procedimiento. De esta manera concluyeron que la complementación de un anestésico local con epinefrina en los procedimientos del pene ofrece ventajas como: alta satisfacción del paciente, infiltración relativamente indolora, baja tasa de complicaciones, mejor visión del campo operatorio y efecto prolongado de los anestésicos, que resulta en disminución del dolor local. Debido a la anatomía del órgano, no existe riesgo de necrosis relacionada con el bloqueo del anillo peneano subcutáneo. Por lo tanto, la idea de que la epinefrina no debe administrase en los procedimientos del pene se considera obsoleta. ${ }^{8}$

La seguridad de este tipo de procedimientos en niños ha demostrado buenos resultados, con excelente eficacia analgésica posoperatoria y disminución considerable de los síntomas sistémicos relacionados con la anestesia general. ${ }^{9-11}$ Algunos estudios señalan casos aislados de intoxicación por lidocaína, convulsiones e insuficiencia respiratoria en pacientes pediátricos. ${ }^{12-14}$ Los autores recomiendan no administrar la dosis máxima permitida en niños y aplicar la dosis más baja, diluida en solución fisiológica.

\section{Técnica de bloqueo del pene}

El pene se encuentra inervado por el nervio pudendo (s2-s4). Posteriormente, éste se divide en los nervios dorsales derecho e izquierdo, que pasan por debajo de la sínfisis del pubis y viajan por debajo de la fascia de Buck para aportar la inervación sensorial del pene. La Figura 1 muestra un corte transversal de la anatomía del pene.

Antes de la infiltración con anestesia parenteral, se recomienda la aplicación de un anestésico tópico local (EMLA), 45 minutos antes del procedimiento. ${ }^{15,16}$ En caso de sospecha de torsión testicular o infección de la piel donde se realizará la punción, se contraindica este procedimiento.

Para efectuar el procedimiento se coloca al paciente en posición supina con los genitales expuestos, se realiza asepsia y antisepsia, y se colocan campos estériles. Posteriormente se administra analgesia parenteral o sedación endovenosa.

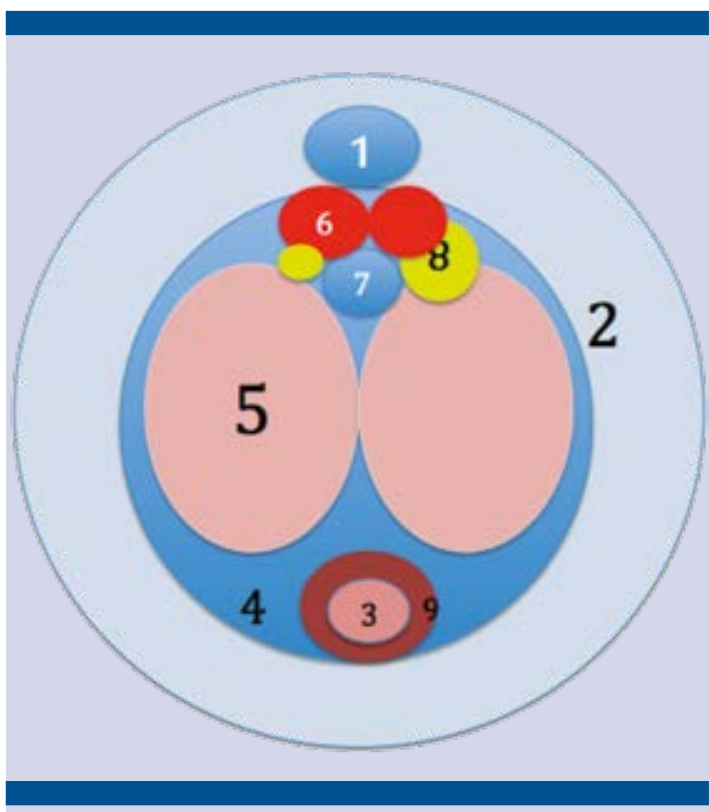

Figura 1. Anatomía transversal del pene: vena dorsal del pene (1), piel (2), uretra (3), fascia de Buck (4), cuerpo cavernoso (5), arteria dorsal (6), vena dorsal profunda (7), nervio dorsal del pene (8) y cuerpo esponjoso (9). 
Entre los diferentes tipos de bloqueo regional del pene se encuentran:

1. Infiltración local (se implementa para remover lesiones pequeñas, como verrugas genitales). El anestésico se aplica en ambos lados de la lesión, con la aguja puncionando la piel, $2 \mathrm{~cm}$ por debajo de la lesión y se infiltran 2-3 mL de lidocaína en forma de abanico.

2. Infiltración local del prepucio distal. Se considera la técnica de elección para realizar incisiones en el prepucio distal, para el tratamiento de la parafimosis. En esta técnica se introduce una aguja de 27-GA, a las 12 de la carátula del reloj, sobre la base del prepucio, avanzando distalmente e infiltrando el anestésico (2-3 $\mathrm{mL}$ de lidocaína).

3. Infiltración local para bloqueo circunferencial. También se utiliza como técnica de referencia antes de la corrección de la parafimosis. Se introduce una aguja de 27-GA y se divide la base del pene en cuadrantes, con la finalidad de infiltrar tres fracciones del anestésico por vía subcutánea en cada uno (se aplican 1-2 mL en cada cuadrante).

4. Bloqueo del pene. Es la técnica de elección para efectuar la circuncisión, reparación de la enfermedad de Peyronie, meatotomía e inserción de prótesis, incluso puede acompañarse de las técnicas previamente descritas. Los nervios dorsales derecho e izquierdo deben bloquearse tan proximal a la base del pene como sea posible. Para esta técnica se introduce una aguja de 27-GA, a las 2 y 10 de la carátula del reloj, y en cada punto se dirige la aguja de manera medial, a una profundidad de $0.5 \mathrm{~cm}$; la pérdida de la resistencia indica que se encuentra en la fascia de
Buck. Una vez logrado esto, se realiza una aspiración para descartar que no se haya entrado en alguna de las estructuras vasculares que viajan adyacentes a los nervios y se infiltran 2-4 mL del anestésico en cada lado. Otra forma de lograr este bloqueo es infiltrar 2-4 mL en cada lado de la vena dorsal superficial, con cuidado de no puncionarla (Figura 2).

\section{Bloqueo inguino-escrotal y del cordón espermático}

Los procedimientos que se realizan con anestesia local incluyen: reparación de hernia inguinal,

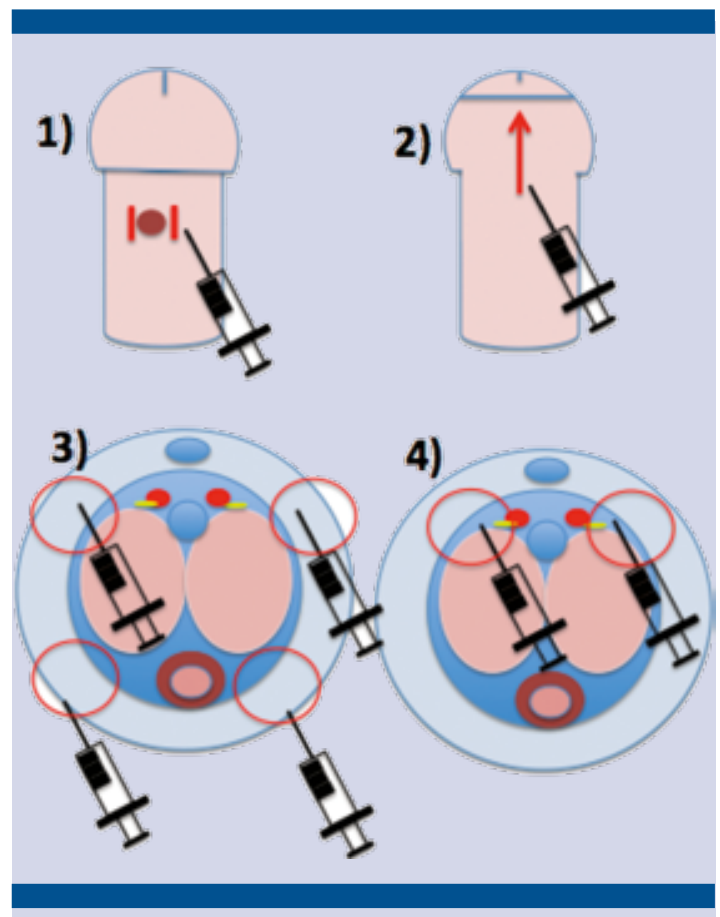

Figura 2. Técnicas de infiltración del pene. 1) Infiltración local (se indica para remover lesiones pequeñas, como verrugas genitales). 2) Infiltración local del prepucio distal (incisiones en el prepucio distal para el tratamiento de la parafimosis). 3) Infiltración local para bloqueo circunferencial (reducción de parafimosis.) 4) Bloqueo del pene (circuncisión, reparación de enfermedad de Peyronie, meatotomía e inserción de prótesis de pene). 
biopsia de ganglio inguinal, hidrocelectomía, biopsia testicular, orquidopexia, orquiectomía y exploración escrotal. ${ }^{17}$ (Cuadro 3).

Estos procedimientos pueden efectuarse con bloqueo inguinal o del cordón espermático, con o sin sedación. Un estudio en pacientes africanos no reportó complicaciones secundarias con esta técnica. En ese estudio, 97\% de los casos recibieron tratamiento ambulatorio y egresaron el mismo día del procedimiento. ${ }^{17}$ Esta técnica de bloqueo regional también proporciona excelentes resultados cuando se combina con anestesia general, lo que resulta en mejor grado de analgesia postoperatoria. ${ }^{18}$

Marchal y su grupo evaluaron la aplicación de bloqueo inguinal con infiltración local para hidrocelectomía y resección de quistes del epidídimo. Entre sus resultados reportaron 94\% de los casos con excelente tolerancia al procedimiento, $2 \%$ con buena reacción y $4 \%$ sin reacción al bloqueo regional. Los principales efectos adversos fueron: cólico renoureteral, hematoma escrotal y dehiscencia de la herida, que suponen complicaciones intrínsecas al procedimiento y no se relacionan con la técnica anestésica. Los autores concluyeron que el bloqueo inguinal es una técnica útil y no provoca efectos adversos. ${ }^{19}$

Cuadro 3. Procedimientos en los que se aplica anestesia inguinal y del cordón espermático con éxito

\begin{tabular}{|c|c|}
\hline Procedimiento & Tipo de anestesia \\
\hline $\begin{array}{l}\text { Hidrocelectomía } \\
\text { Quiste de } \\
\text { epidídimo }\end{array}$ & $\begin{array}{l}\text { Bloqueo del cordón y anestesia local } \\
\text { Bloqueo del cordón y anestesia local }\end{array}$ \\
\hline Vasectomía & Bloqueo del cordón y anestesia local \\
\hline Varicocelectomía & Bloqueo del cordón y anestesia local \\
\hline Biopsia testicular & Bloqueo del cordón y anestesia local \\
\hline Orquiectomía & $\begin{array}{l}\text { Bloqueo inguinal, de cordón y anes- } \\
\text { tesia local }\end{array}$ \\
\hline Orquidopexia & Bloqueo del cordón y anestesia local \\
\hline
\end{tabular}

El bloqueo del cordón espermático con infiltración local en pacientes intervenidos para vasectomía ha reportado analgesia altamente satisfactoria, lo que permite al paciente egresar pocas horas después del procedimiento, sin exponerlo a los riesgos de la anestesia general $y$ del bloqueo espinal. ${ }^{20}$

La varicocelectomía subinguinal con microscopio es una técnica segura cuando se realiza con anestesia regional, con bloqueo inguinal e infiltración local; entre sus ventajas se señalan menor recurrencia e índice de formación de hidrocele, y mayor tasa de embarazos espontáneos. ${ }^{21}$ También se ha descrito el bloqueo del cordón espermático en pacientes con varicocelectomía inguinal, mediante la aplicación de 5-10 mL de lidocaína al $0.8 \%{ }^{22}$

Fhamy y sus colaboradores describieron un grupo de pacientes a quienes se tomó biopsia testicular, en quienes aplicaron lidocaína en el escroto, la fascia de dartos y la túnica albugínea, además de la infiltración de $1 \mathrm{~mL}$ de lidocaína en el testículo. Con esta técnica de anestesia local se logró una buena analgesia, sin reportar efectos adversos. ${ }^{23}$

Diversos estudios han reportado excelentes resultados con el bloqueo inguinal y la infiltración local de lidocaína para orquiectomías simples subcapsulares. Una serie de 100 pacientes con orquiectomía subcapsular evidenció buena tolerancia con esta técnica anestésica. La orquiectomía radical, efectuada en pacientes con cáncer testicular, puede efectuarse con bloqueo inguinal; sin embargo, aumenta significativamente el grado de dificultad para el cirujano, porque no existe adecuada relajación de los músculos de la pared abdominal. ${ }^{24}$

\section{Técnica de boqueo inguinal}

El escroto se encuentra inervado por los nervios escrotales posteriores y la rama perineal del 
nervio pudendo (S2-S4); de forma anterolateral por la rama genital del nervio genitofemoral (L1L2), anterior por los nervios escrotales anteriores (nervio ilioinguinal) e inferior por las ramas perineales del nervio cutaneofemoral posterior (S2-S3) (Figura 3). Este bloqueo se indica en procedimientos como: orquiectomía, biopsia de ganglio y plastia inguinales; hidrocelectomía, criptorquidia y orquidopexia.

El procedimiento se inicia con la infiltración del tejido subcutáneo donde se realizará la incisión; posteriormente se palpa la espina iliaca superior y se localiza un punto, $2 \mathrm{~cm}$ medial a ésta, para insertar una aguja de 21-GA, de manera perpendicular a la piel hasta perder la resistencia o percibir un pequeño desgarro, que ocurre al pasar la aponeurosis del oblicuo mayor; se realiza la aspiración (con la finalidad de no dañar algún vaso) y en caso de ser negativa, se introduce una cuarta parte del anestésico, para de esta manera bloquear el nervio iliohipogástrico. Después se avanza la aguja en la misma dirección y $0.5 \mathrm{~cm}$

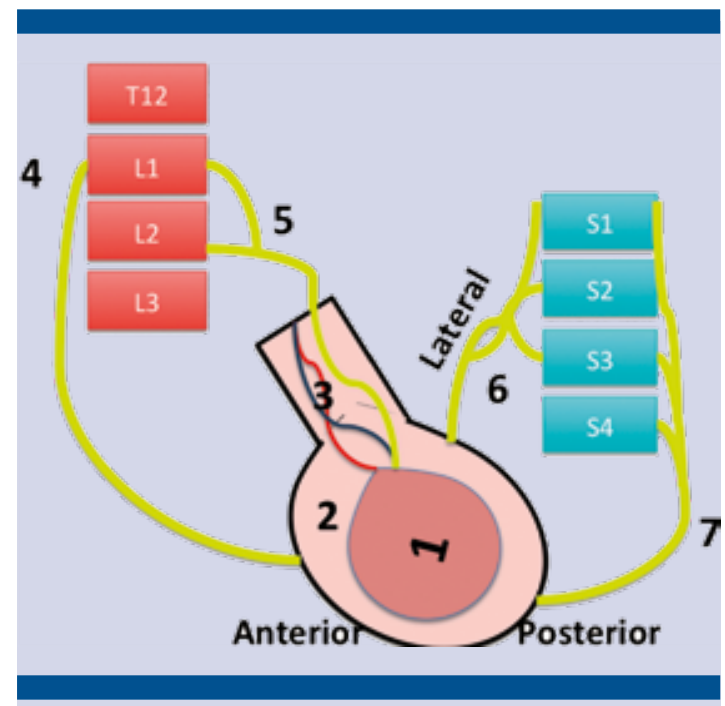

Figura 3. Inervación del escroto y testículo. 1) Testículo, 2) escroto, 3) cordón espermático, 4) nervio ilioinguinal, 5) nervio genitofemoral, 6) rama perineal del nervio cutáneo femoral posterior y 7) nervio pudendo. debajo, entre el oblicuo interno y el transversal abdominal se aplica otra cuarta parte del anestésico para bloquear el nervio ilioinguinal, $y$, finalmente, se introduce la aguja $0.5 \mathrm{~cm}$ más y se aplica la última fracción del anestésico para bloquear el nervio cutáneo. Posteriormente se palpa el orificio inguinal profundo $(1-1.5 \mathrm{~cm}$ por encima del ligamento inguinal, en su porción media), se introduce la aguja en el canal y se aspira, que de no obtener sangre se infiltran $5 \mathrm{~mL}$ de anestesia para bloquear el nervio genitofemoral. Finalmente, se retira la aguja y se infiltran $2 \mathrm{~cm}$ por encima y lateral del tubérculo púbico para bloquear cualquier inervación contralateral (Figura 4).

\section{Bloqueo del cordón espermático}

El cordón espermático suministra la irrigación arterial, el drenaje venoso y linfático, y la iner-

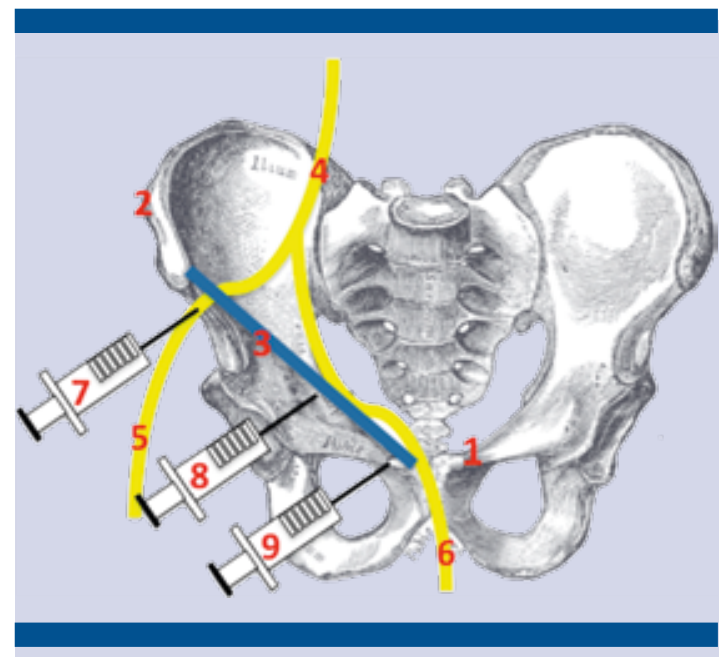

Figura 4. Anatomía y técnica de bloqueo inguinal. 1) Tubérculo del pubis, 2) espina iliaca antero-superior, 3) ligamento inguinal, 4) nervio genitofemoral, 5) rama femoral del nervio genitofemoral, 6) rama genital del nervio genitofemoral, 7) punto de acceso para bloquear el nervio iliohipogástrico, ilioinguinal y la rama femoral del nervio genitofemoral, 8) punto de acceso para bloquear el nervio ilioinguinal y genitofemoral, 9) punto de acceso para bloquear la rama genital del nervio genitofemoral y las ramas contralaterales. 
vación sensitiva y autónoma hacia el testículo. Los nervios que viajan en su interior son el nervio cremastérico (rama genital del nervio genitofemoral) y los nervios testiculares (Figura 5).

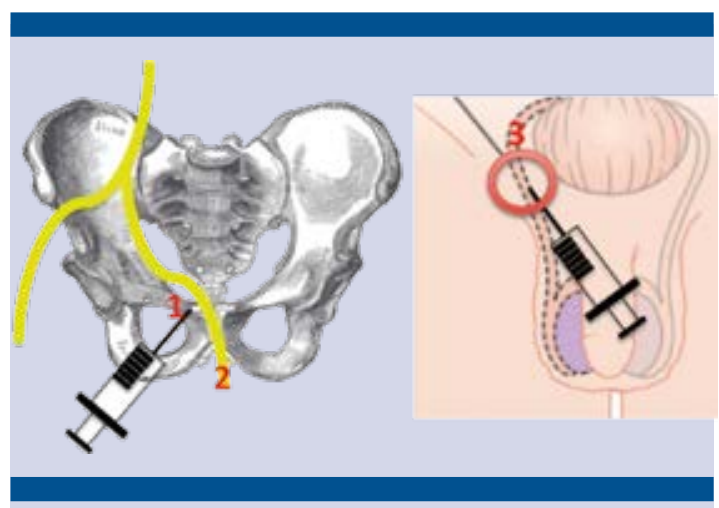

Figura 5. Anatomía y técnica de bloqueo del cordón espermático. 1) tubérculo del pubis, 2) rama genital del nervio genitofemoral y 3) cordón espermático.

Este bloqueo se indica en casos de hidrocelectomía, vasectomía, varicocelectomía, biopsia testicular, orquiectomía escrotal y orquidopexia, siempre acompañados de infiltración local en el escroto, en el sitio donde se realizará la incisión.

El primer paso consiste en infiltrar por vía subcutánea la línea donde se realizará la incisión; posteriormente se identifica el tubérculo del pubis, se introduce una aguja de 21-GA, $1 \mathrm{~cm}$ por debajo y medial al tubérculo del pubis hasta alcanzar el hueso; se aspira para verificar que no se encuentre en un vaso y se infiltran tres cuartas partes de la dosis, dejando una cuarta parte en la jeringa, en caso de requerirse durante el procedimiento.

Otra manera de bloquear el cordón consiste en infiltrar el tejido subcutáneo donde se realizará la incisión; después debe percibirse el cordón, efectuar la tracción del testículo de manera podálica e introducir la aguja de forma lateral al cordón espermático, en dirección al orificio inguinal externo $4 \mathrm{~cm}$ por arriba del testículo; se aspira para verificar que no se encuentre en un vaso y se infiltra el anestésico a las 9, 12 y 3 de la caratula del reloj, siempre con previa aspiración al aplicarlo.

\section{REFERENCIAS}

1. Soh CR, Ng SB, Lim SL. Dorsal penile nerve block. Paediatr Anaesth. 2003;13(4):329-33.

2. Taddio A, Pollock N, Gilbert-MacLeod C, Ohlsson K, Koren G. Combined analgesia and local anesthesia to minimize pain during circumcision. Arch Pediatr Adolesc Med. 2000;154(6):620-3.

3. Garry DJ, Swoboda E, Elimian A, Figueroa R. A video study of pain relief during newborn male circumcision. J Perinatol. 2006;26(2):106-10.

4. Hsu GL, Hsieh CH, Chen HS, Ling PY, Wen HS, Liu LJ, Chen $\mathrm{CW}$, Chua $\mathrm{C}$. The advancement of pure local anesthesia for penile surgeries: can an outpatient basis be sustainable? J Androl 2007;28(1):200-5.

5. Hsu GL, Hsieh $\mathrm{CH}$, Wen HS, et al. Outpatient penile implantation with the patient under a novel method of crural block. Int J Androl. 2004;27(3):147-51.

6. Hsu GL, Hsieh $\mathrm{CH}$, Wen HS, et al. Outpatient surgery for penile venous patch with the patient under local anesthesia. J Androl. 2003;24(1);35-9.

7. Malkoc E, Ates F, Uguz S, et al. Effective penile block for circumcision in adults. Wien Klin Wochenschr. 2012;124(1314):434-8.

8. Schnabl SM, Herrmann N, Wilder D, et al. Clinical results for use of local anesthesia with epinephrine in penile nerve block. J Dtsch Dermatol Ges. 2014;12(4):332-9.

9. Panda A, Bajwa SJ, Parmer SS. Penile block for paediatric urological surgery: A comparative evaluation with general anaesthesia. Indian J Urol 2011;27(4):457-64.

10. Dalens B1, Vanneuville G, Dechelotte P. Penile block via the subpubic space in 100 children. Anesth Analg. 1989;69(1):41-45.

11. Ovalle A, López PJ, Guelfand M, Zubieta R. Neonatal circumcision with local anesthesia. Results of a standardized protocol. Rev Chil Pediatr. 2016;87(3):175-9.

12. Heiberg IL, Nebrich L, Pedersen P. Seizures and respiratory failure following local anaesthesia administered for circumcision. Ugeskr Laeger. 2015;177(27):v11140641.

13. Doye E, Desgranges FP, Stamm D, et al. Severe local anesthetic intoxication in an infant undergoing circumcision. Arch Pediatr. 2015;22(3):303-5.

14. Özer AB, Erhan ÖL. Systemic toxicity to local anesthesia in an infant undergoing circumcision. Agri. 2014;26(1):43-46.

15. Lehr VT, Cepeda E, Frattarelli DA, et al. Lidocaine $4 \%$ cream compared with lidocaine $2.5 \%$ and prilocaine $2.5 \%$ 
or dorsal penile block for circumcision. Am J Perinatol. 2005;22(5):231-7.

16. Choi WY, Irwin MG, Hui TW, et al. EMLA cream versus dorsal penile nerve block for postcircumcision analgesia in children. Anesth Analg. 2003;96(2):396-9.

17. Magoha GA. Local infiltration and spermatic cord block for inguinal, scrotal and testicular surgery. East Afr Med J. 1998;75(10):579-81.

18. Burden RJ1, O'Kelly SW, Sutton D, et al. Spermatic-cord block improves analgesia for day-case testicular surgery. Br J Urol. 1997;80(3):472-5.

19. Marchal Escalona C1, Chicharro Molero JA, Martín Morales $A$, et al. Local anesthesia in the surgical management of hydrocele and cysts of the spermatic cord. Actas Urol Esp. 1993;17(1):68-72.
20. Shih $\mathrm{G}$, Njoya M, Lessard $\mathrm{M}$, et al. Minimizing pain during vasectomy: the mini-needle anesthetic technique J Urol. 2010;183(5):1959-63.

21. Çayan S, Shavakhabov S, Kadioğlu A. Treatment of palpable varicocele in infertile men: a meta-analysis to define the best technique. J Androl. 2009;30:33-40.

22. Hsu GL, Ling PY, Hsieh $\mathrm{CH}$, et al. Outpatient varicocelectomy performed under local anesthesia. Asian J Androl. 2005;7(4):439-44.

23. Fahmy I, Kamal A, Aboulghar M, et al. Percutaneous aspiration biopsy using an intravenous catheter for testicular sperm retrieval in patients with obstructive azoospermia. Reprod Biomed Online. 2004;9(1):102-5.

24. Kiesling VJ, Schroeder DE, Pauljev $P$, et al. Spermatic cord block and manual reduction: primary treatment for spermatic cord torsion. J Urol. 1984;132(5):921-3.

\section{AVISO PARA LOS AUTORES}

Revista Mexicana de Urología tiene una nueva plataforma de gestión para envío de artículos: https://www.revisionporpares.com/index.php/RMUrol ahí podrá inscribirse a la base de datos administrada por el sistema Open Journal System (OJS) que ofrece las siguientes ventajas para los autores:

- Subir sus artículos directamente al sistema.

- Conocer, en cualquier momento, el estado de los artículos enviados, es decir, si ya fueron asignados a un revisor, aceptados con o sin cambios, o rechazados.

- Participar en el proceso editorial corrigiendo y modificando sus artículos hasta su aceptación final. 